Supplementary Information

\title{
Contrast-Enhanced Multispectral Photoacoustic Imaging for Irregular Hepatectomy Navigation: a Pilot Study
}

Yueming Zhang ${ }^{1, \#, ~ J i n g ~} L v^{2}, \#$, Pingguo Liu ${ }^{3}$, Xingyang Zhao ${ }^{1}$, Kang Chen ${ }^{1}$, Qiaolin Li1, Liming Nie ${ }^{2}$, , and Chihua Fang ${ }^{1}$ *

1. Department of Hepatobiliary Surgery, Zhujiang Hospital, Southern Medical University, Guangzhou, China.

2. State Key Laboratory of Molecular Vaccinology and Molecular Diagnosis \& Center for Molecular Imaging and Translational Medicine, School of Public Health, Xiamen University, Xiamen, China.

3. Department of Hepatobiliary Surgery, Zhongshan Hospital Xiamen

University, Xiamen, China.

\#These authors contributed equally to this work.

*Parallel corresponding authors. 
SI Figures, Tables, and Schemes

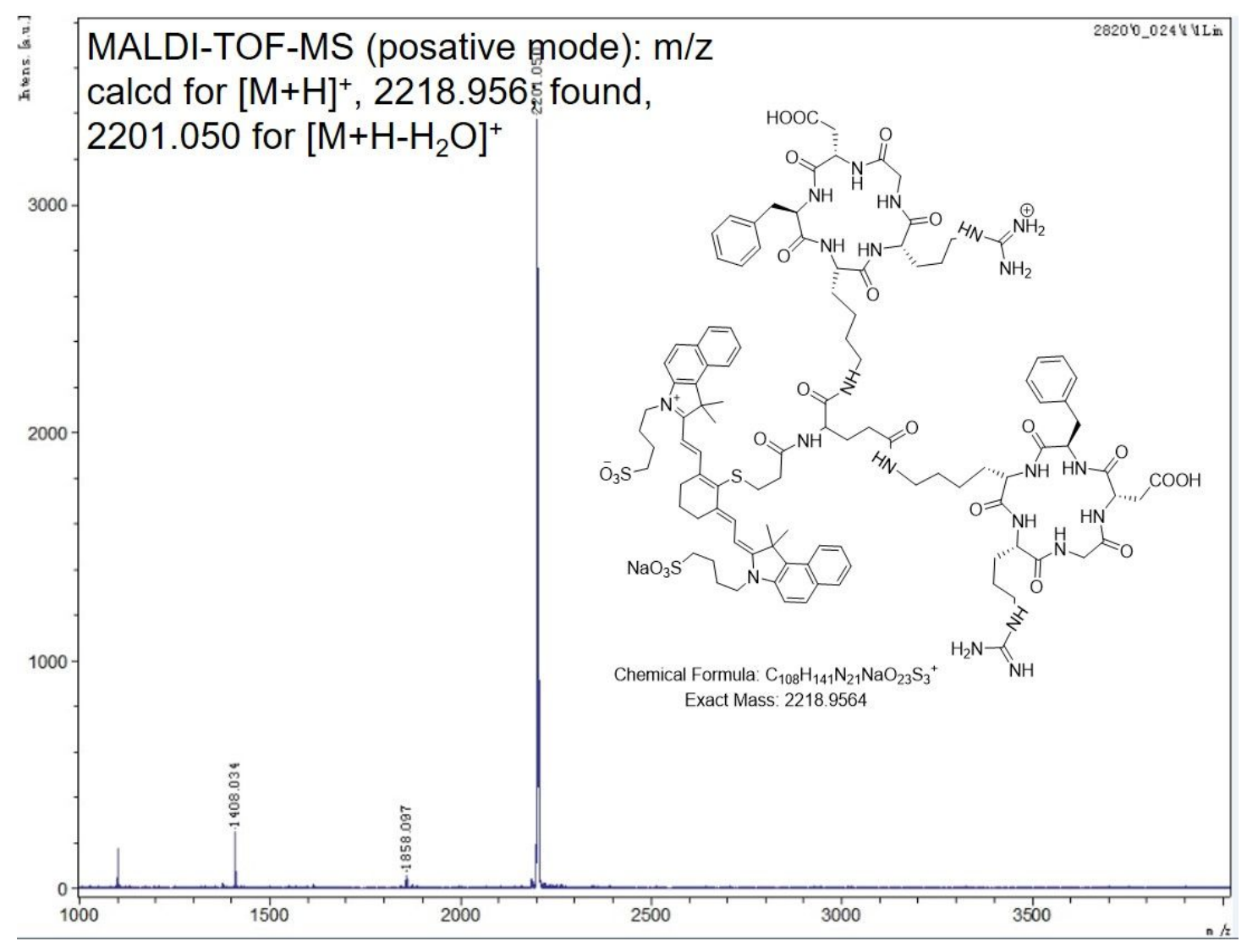

SI Figure 1: High-resolution mass spectrum of IR820-E[c(RGDfK) $]_{2}$. 




b

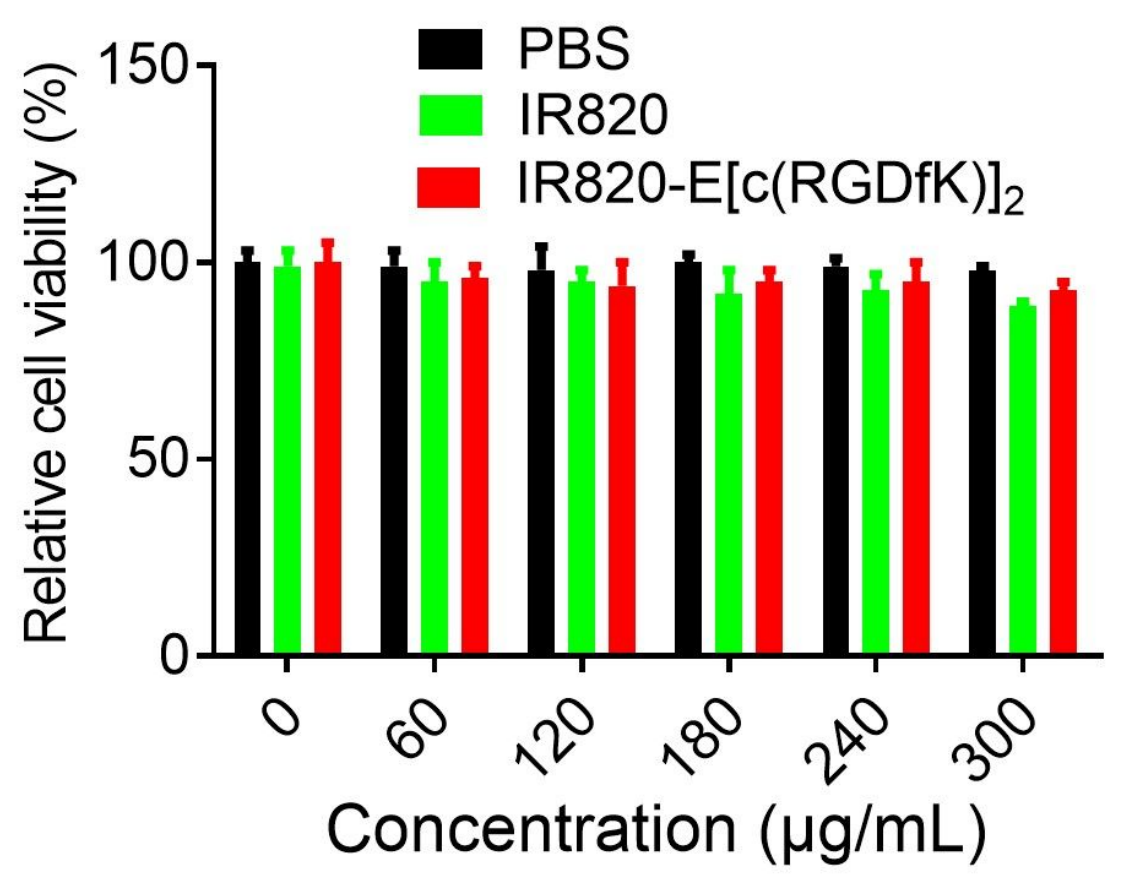

SI Figure 2: (a) Confocal microscopy of Hep-G2 cells stained with PBS, IR820$\mathrm{E}[\mathrm{c}(\mathrm{RGDfK})]_{2}$ and IR820-E[c(RGDfK $\left.)\right]_{2}$ with blocking $(\mathrm{n}=3)$. Nuclear is stained with 
DAPI (a blue dye). (b) The viability of Hep-G2 cells treated with PBS, IR820 and $\operatorname{IR} 820-E[c(R G D f K)]_{2}$ at various concentration $(n=3)$.

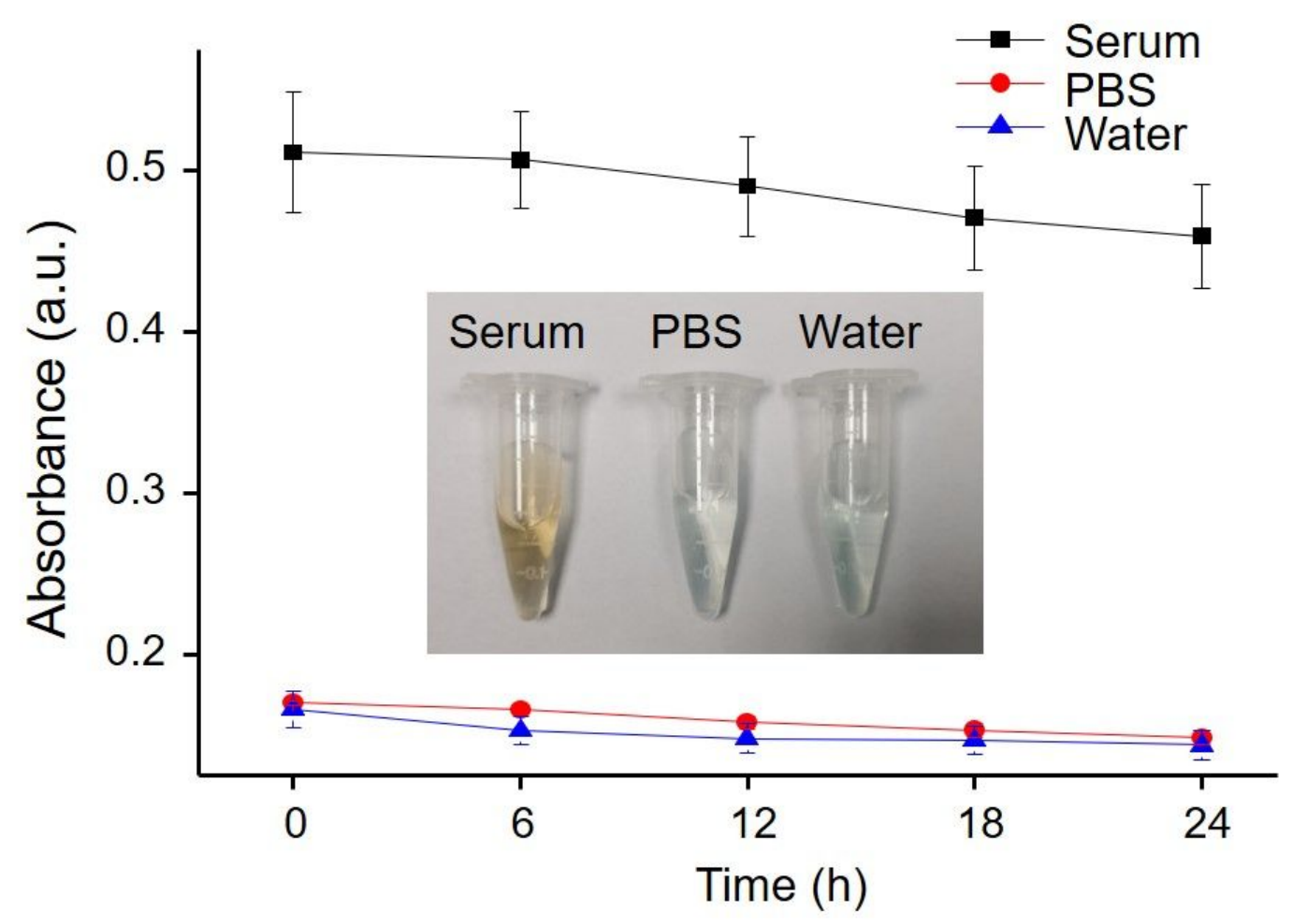

SI Figure 3: The absorbance values of IR820-E[c(RGDfK) $]_{2}$ at different time points in serum, PBS, and distilled water. 
a

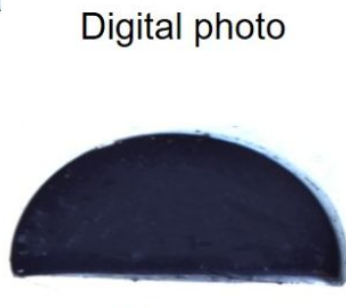

$1 \mathrm{~mm}$
Binarize



NB

b

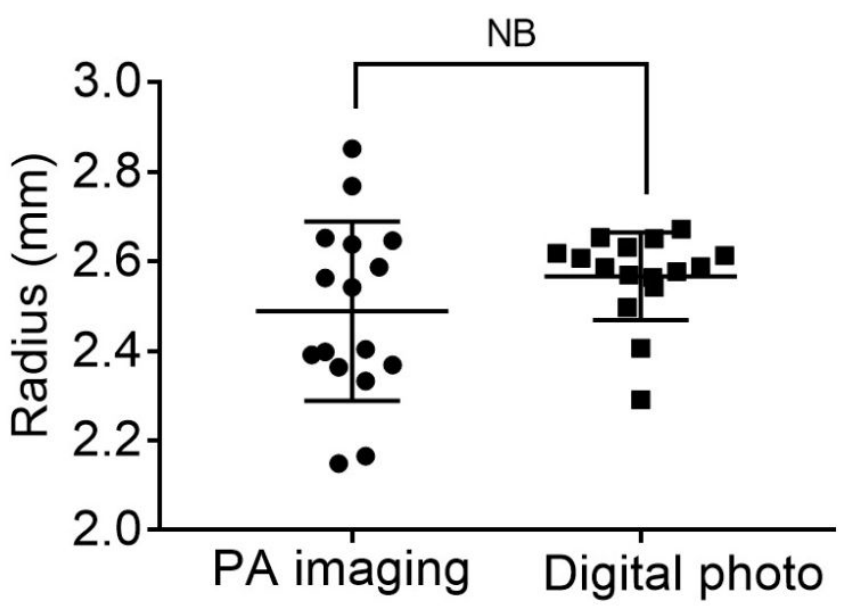

SI Figure 4: The phantom study for optimizing imaging capability of the experimental setup in this study. (a) Representative digital photo, binarized image and PA imaging of gelatin inclusions containing black ink. (b) Quantitative analysis the radius of phantom from digital photo and PA imaging. The results showed no statistical 
significance, which demonstrated the capability of the experimental setup for delineating tumor boundaries.

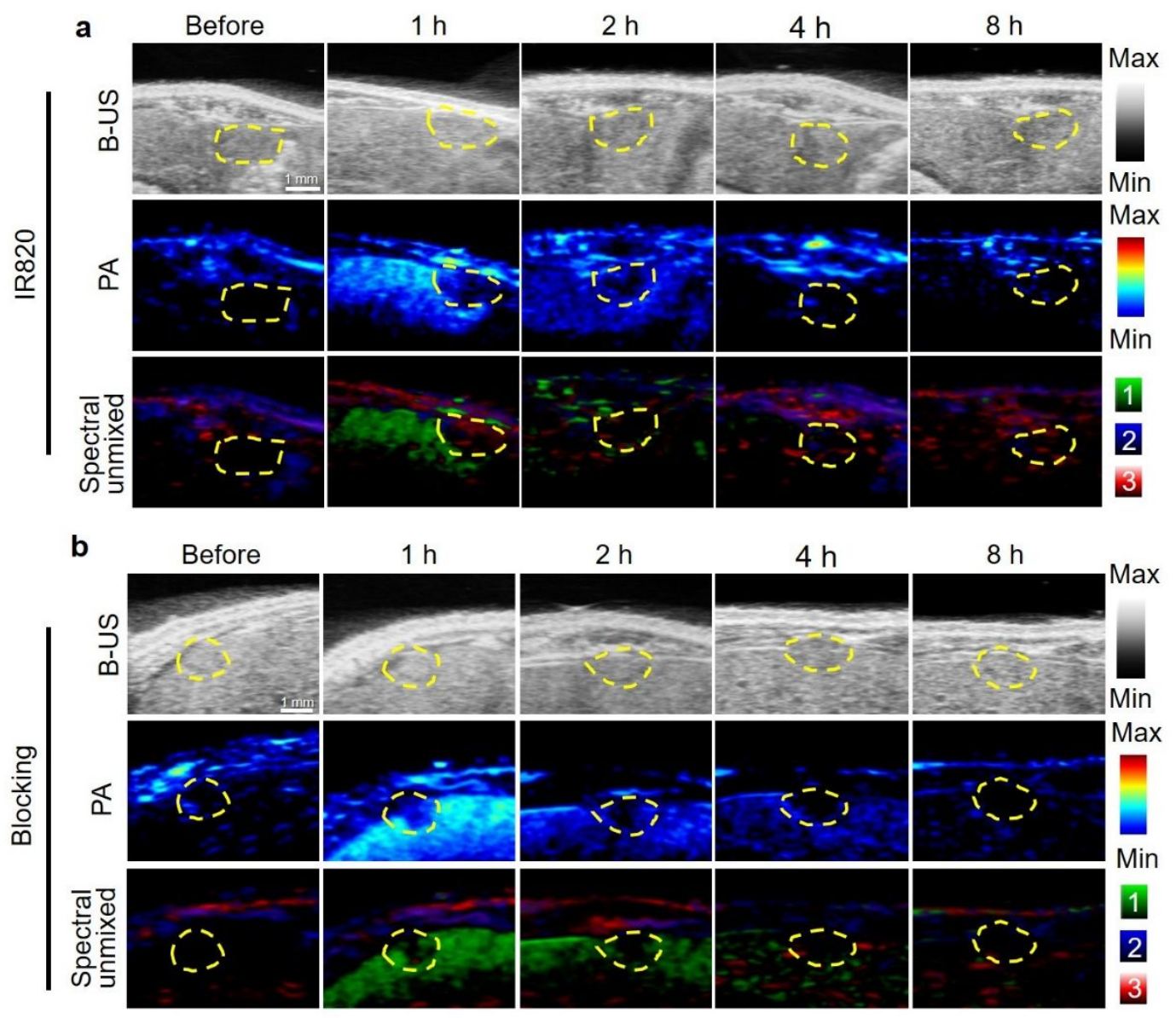


SI Figure 5: Representative B-mode ultrasound and multispectral PA imaging of orthotopic HCC tumor before and after intravenous administration of IR820 (a) and IR820-E[c(RGDfK) $]_{2}$ with blockade (b) $(\mathrm{n}=3)$. 


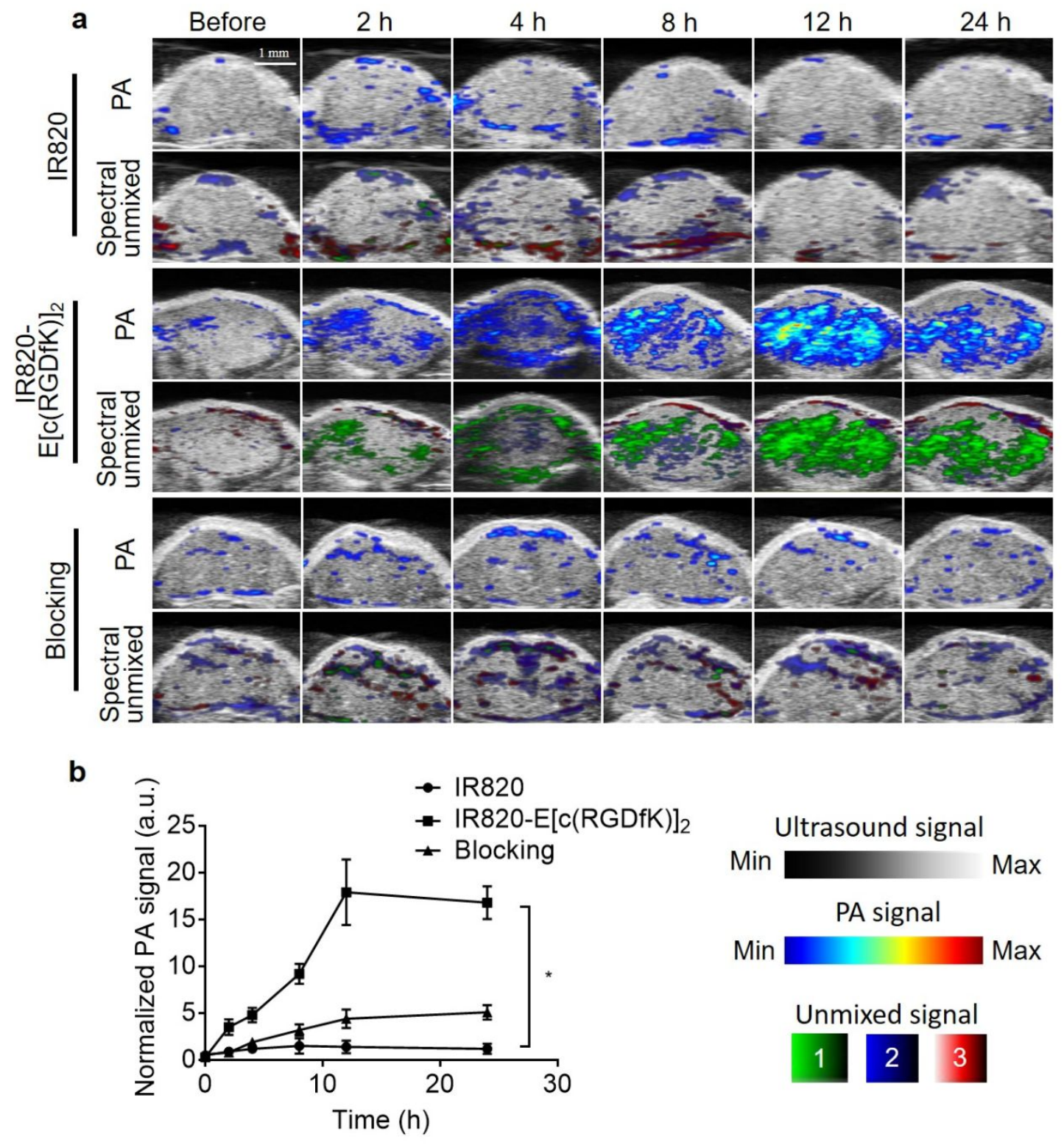

SI Figure 6: (a) Representative B-mode ultrasound and multispectral PA imaging of subcutaneous HCC tumor before and after intravenous administration of IR820, IR820$\mathrm{E}[\mathrm{c}(\mathrm{RGDfK})]_{2}$ and IR820-E[c(RGDfK $\left.)\right]_{2}$ with blockade $(\mathrm{n}=3)$. (b) Quantification of unmixed PA signal of subcutaneous HCC tumor in (a). Values are presented as means \pm SD of 3 animals. $* p<0.05$ vs. IR820. 


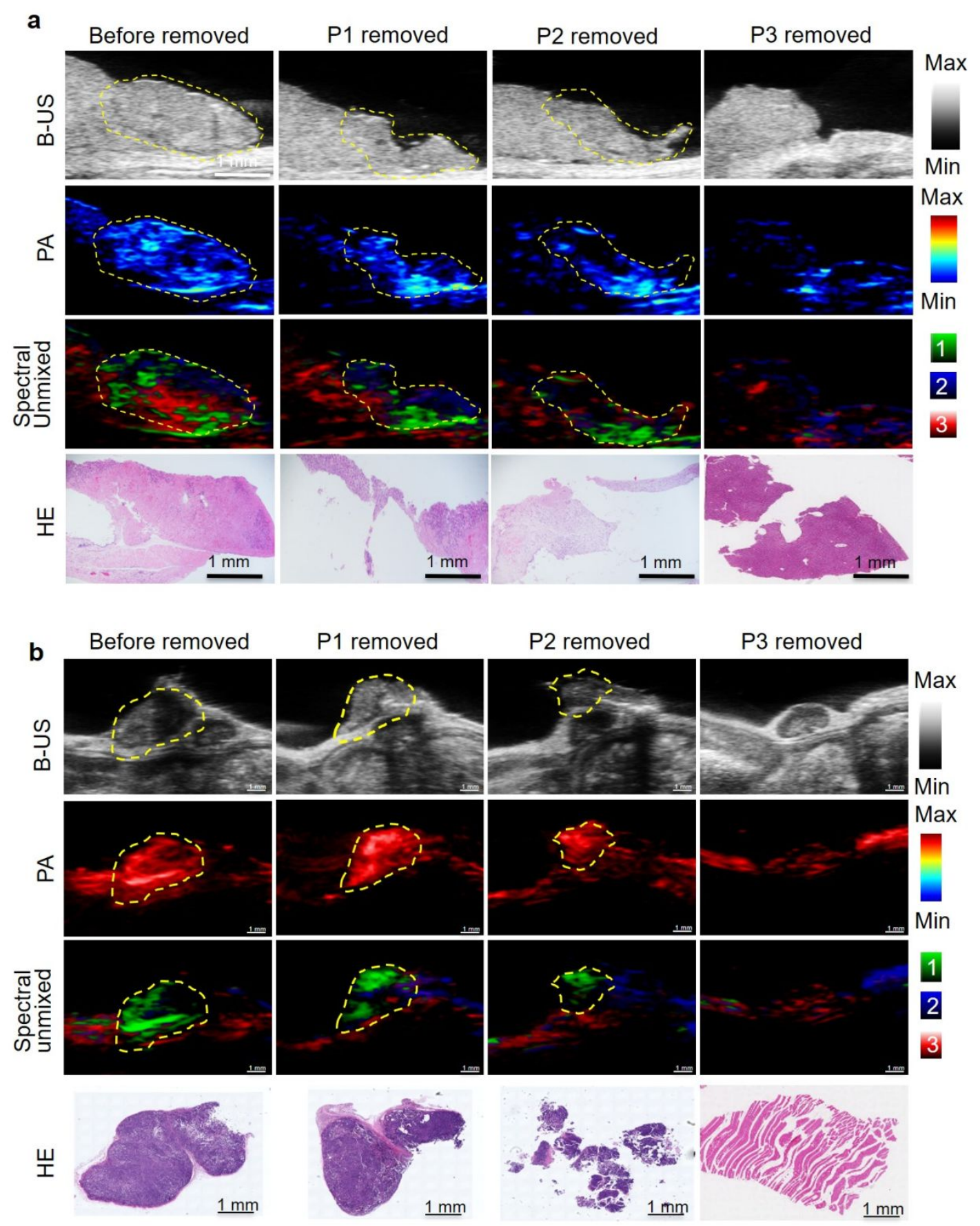

SI Figure 7: (a) Case study 3 of multispectral PA image-guided irregular hepatectomy in orthotopic HCC tumors models. (b) Multispectral PA image-guided irregular hepatectomy in subcutaneous HCC tumors models. 

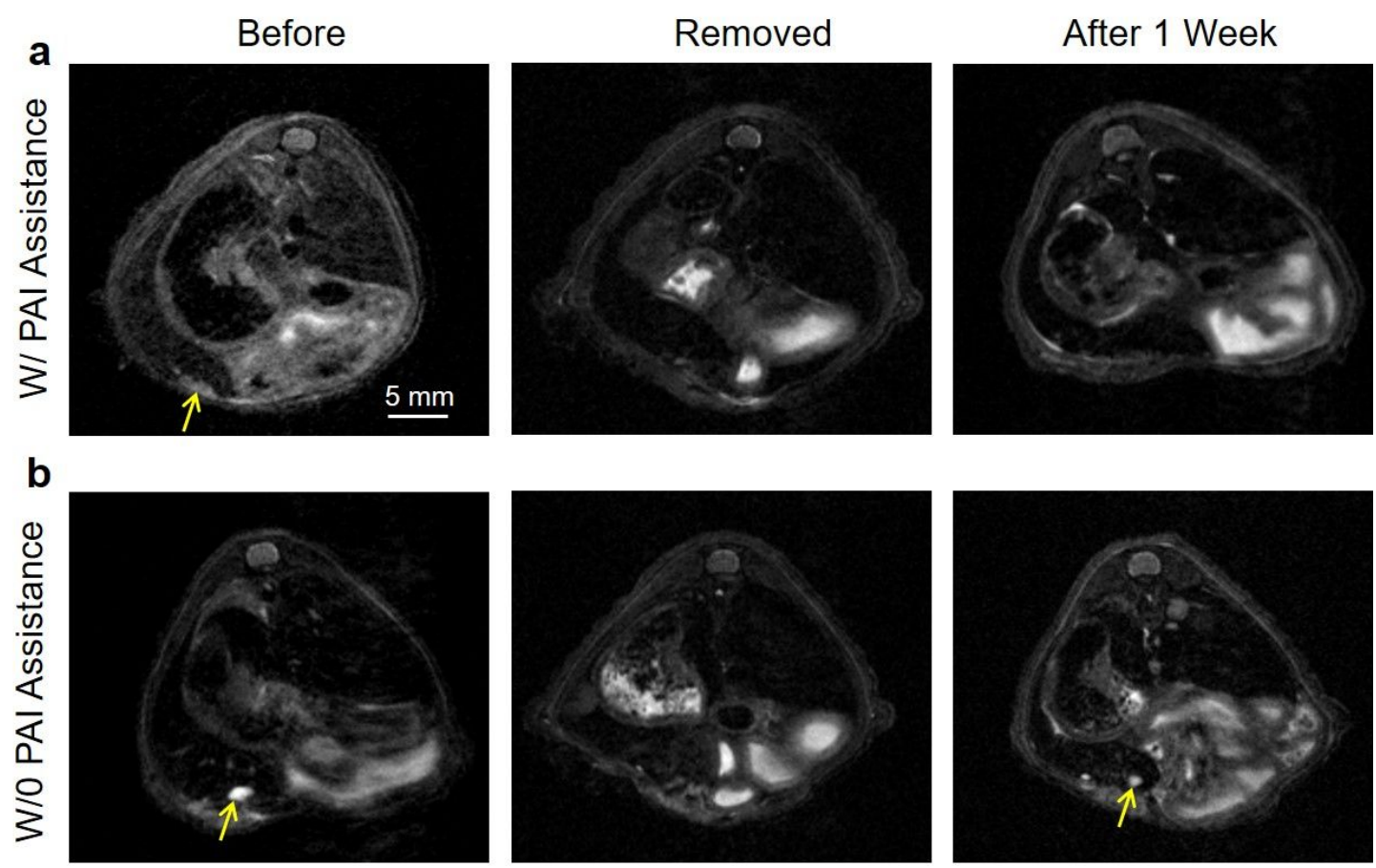

SI Figure 8: (a) T2 magnetic resonance imaging results of tumor regrowth after surgery

with PA imaging assistance after administration of IR820-E[c(RGDfK)]2. (b) T2 magnetic resonance imaging results of tumor regrowth after surgery without PA imaging assistance after administration of IR820-E[c(RGDfK)]2. 

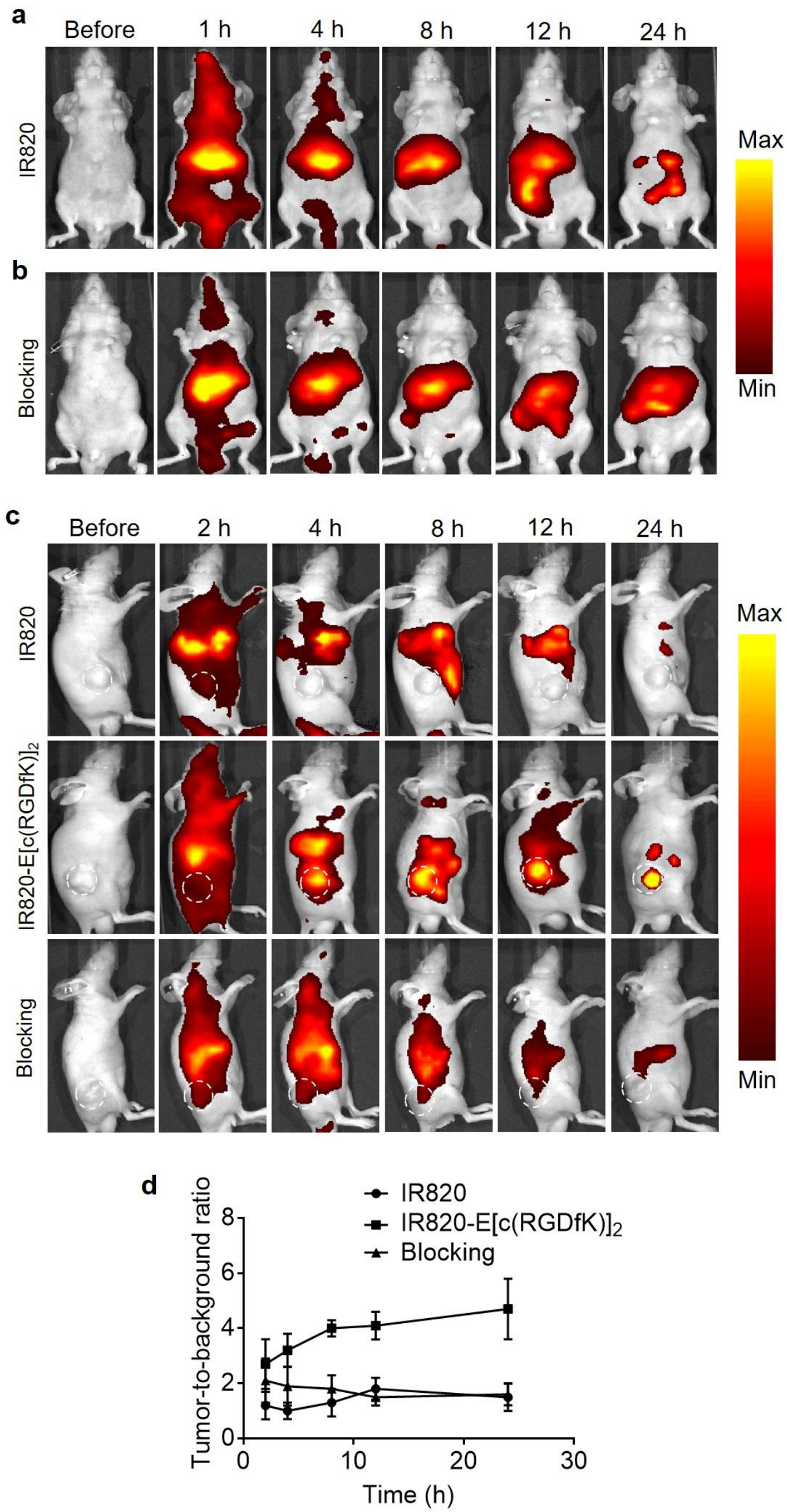
SI Figure 9: (a)-(b) Representative whole-body fluorescent imaging after injection of IR820 and IR820-E[c(RGDfK) $]_{2}$ with blockade in orthotopic HCC tumor models at different time intervals $(\mathrm{n}=3)$. (c) Time-dependent whole-body fluorescent imaging after injection of IR820, IR820-E[c(RGDfK) $]_{2}$ and IR820-E[c(RGDfK) $]_{2}$ with blockade in subcutaneous HCC tumor models $(n=3)$. (d) Fluorescent quantitative analysis of tumor-to-background ratio in (c). 
a

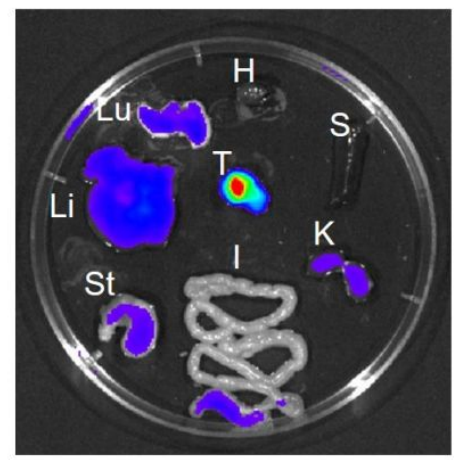

b

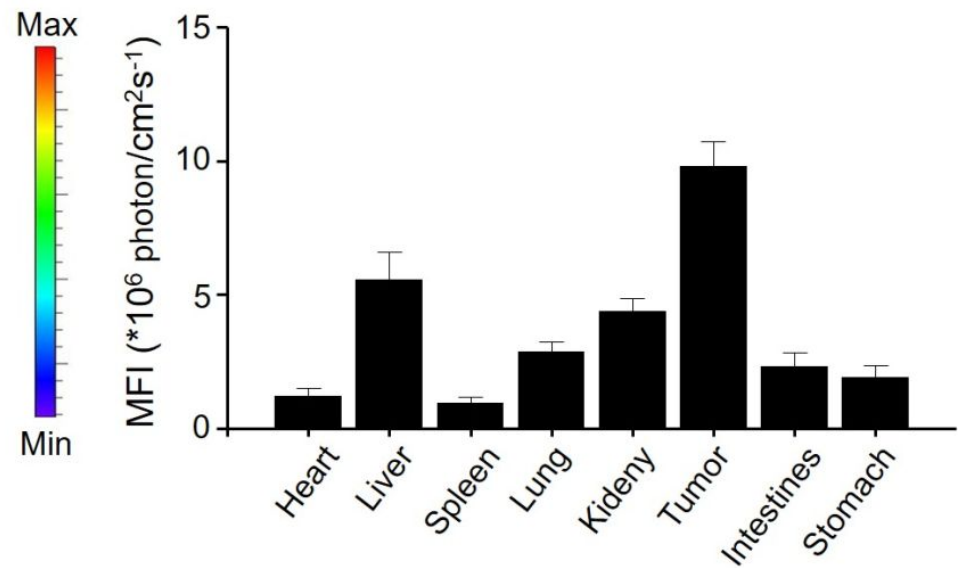

SI Figure 10: (a) Ex vivo NIR imaging of IR820-E[c(RGDfK) $]_{2}$ in main organs and tumor tissues (H, heart; Li, liver; S, spleen; Lu, lung; K, kidney; T, tumor; I, intestines; St, stomach) of tumor bearing mice model. (b) Fluorescence signal analysis of different organs and tumor tissues. 
a \# 8

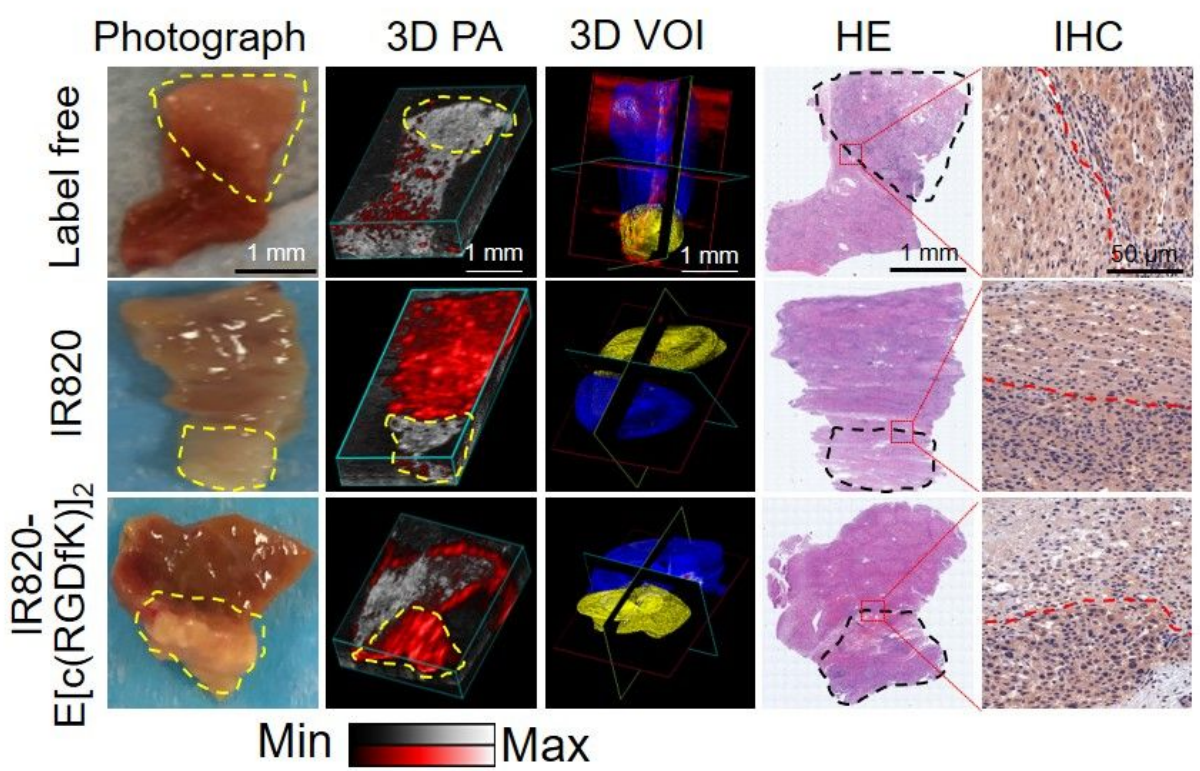

b

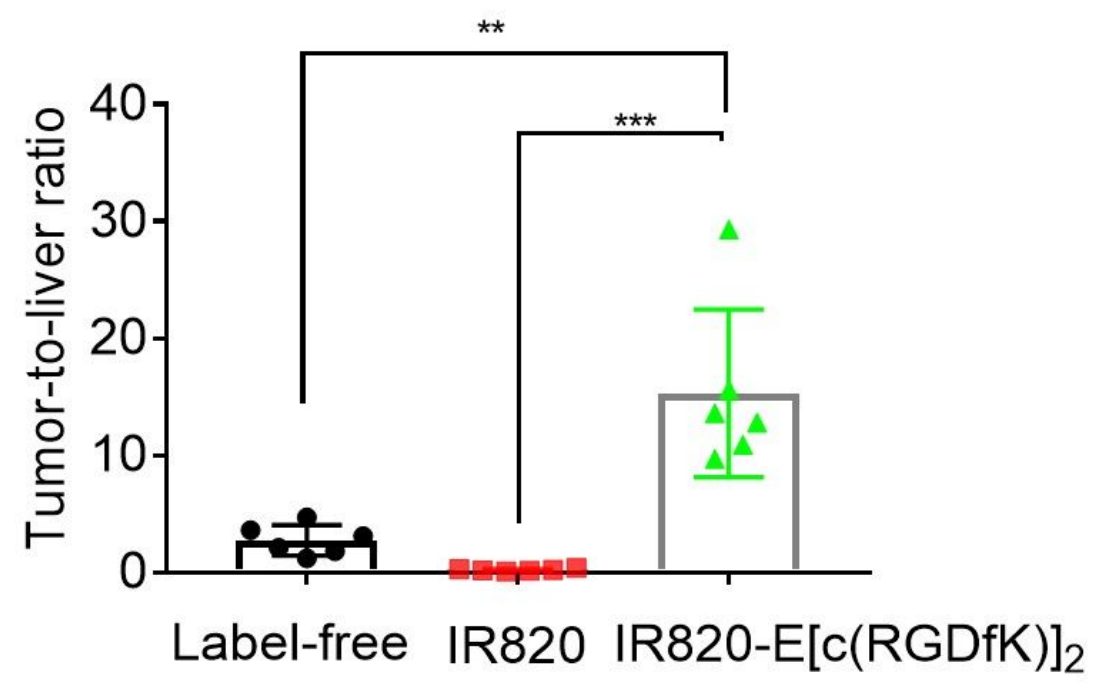

SI Figure 11: (a) Representative 3D PA images and VOI analysis of fresh liver cancer specimens for patient 8 stained with PBS, IR820 and IR820-E[c(RGDfK) $]_{2}$ respectively. The results are in excellent agreement with $\mathrm{H} \& \mathrm{E}$ and $\alpha_{v} \beta_{3}$ IHC staining. Specimens scale bar is $1 \mathrm{~mm}$ (b) Quantitative VOI analysis of photoacoustic signal density in tumors and normal liver tissues in (a) ( $\mathrm{n}=6$ specimens/group). ${ }^{* *} p<0.01$, $* * * p<0.001$. 


\begin{tabular}{|c|c|c|c|c|c|c|c|c|}
\hline $\begin{array}{l}\text { Patient } \\
\text { NO. }\end{array}$ & $\begin{array}{l}\text { Gender } \\
\text { (M/F) }\end{array}$ & $\begin{array}{l}\text { Age } \\
(Y)\end{array}$ & $\begin{array}{c}\text { AFP }>400 \mu g / L \\
(Y / N)\end{array}$ & $\begin{array}{l}\text { Hepatitis } \\
\text { Type }\end{array}$ & $\begin{array}{l}\text { Child-Pugh } \\
\text { class }\end{array}$ & $\begin{array}{l}\text { Parameter } \\
\text { of tumor } \\
\text { (cm) }\end{array}$ & $\begin{array}{l}\text { Segment of } \\
\text { Surgical } \\
\text { Resection } \\
\text { (S1-S8) }\end{array}$ & $\begin{array}{c}\text { Differentiation } \\
\text { Grade of Liver } \\
\text { Cancer }\end{array}$ \\
\hline$\# 1$ & $M$ & 75 & $\mathrm{~N}$ & $(-)$ & A & $2-5$ & S2-S3 & G2 \\
\hline \#2 & $M$ & 50 & $Y$ & Type B & A & $2-5$ & S5-S8 & G2 \\
\hline \#3 & $M$ & 68 & $Y$ & Type B & A & $>5$ & $\mathrm{~S} 2-\mathrm{S} 4$ & G2 \\
\hline$\# 4$ & $M$ & 75 & $Y$ & Type B & A & $>5$ & S2-S4,S5,S8 & G3 \\
\hline \#5 & $M$ & 38 & $\mathrm{~N}$ & Type B & A & $2-5$ & S5-S8 & G2 \\
\hline$\# 6$ & $M$ & 70 & $\mathrm{~N}$ & $(-)$ & A & $2-5$ & S6,S7 & G1 \\
\hline$\# 7$ & $M$ & 72 & $\mathrm{~N}$ & Type B & A & $2-5$ & S5,S8 & G2 \\
\hline$\# 8$ & $M$ & 49 & $\mathrm{Y}$ & Type B & A & $>5$ & S4-S8 & G2 \\
\hline$\# 9$ & $M$ & 60 & $Y$ & $(-)$ & A & $2-5$ & S5-S8 & G2 \\
\hline$\# 10$ & $\mathrm{~F}$ & 60 & $\mathrm{Y}$ & Type B & $A$ & $2-5$ & S5-S8 & G2 \\
\hline \#11 & $\mathrm{F}$ & 73 & Y & Type B & A & $2-5$ & S5,S8 & $\mathrm{G} 2$ \\
\hline$\# 12$ & $M$ & 35 & $Y$ & Type B & A & $2-5$ & S5-S8 & G2 \\
\hline
\end{tabular}

SI Table 1: Patient Characteristics and Surgical Pathologic Analysis of liver cancer. 